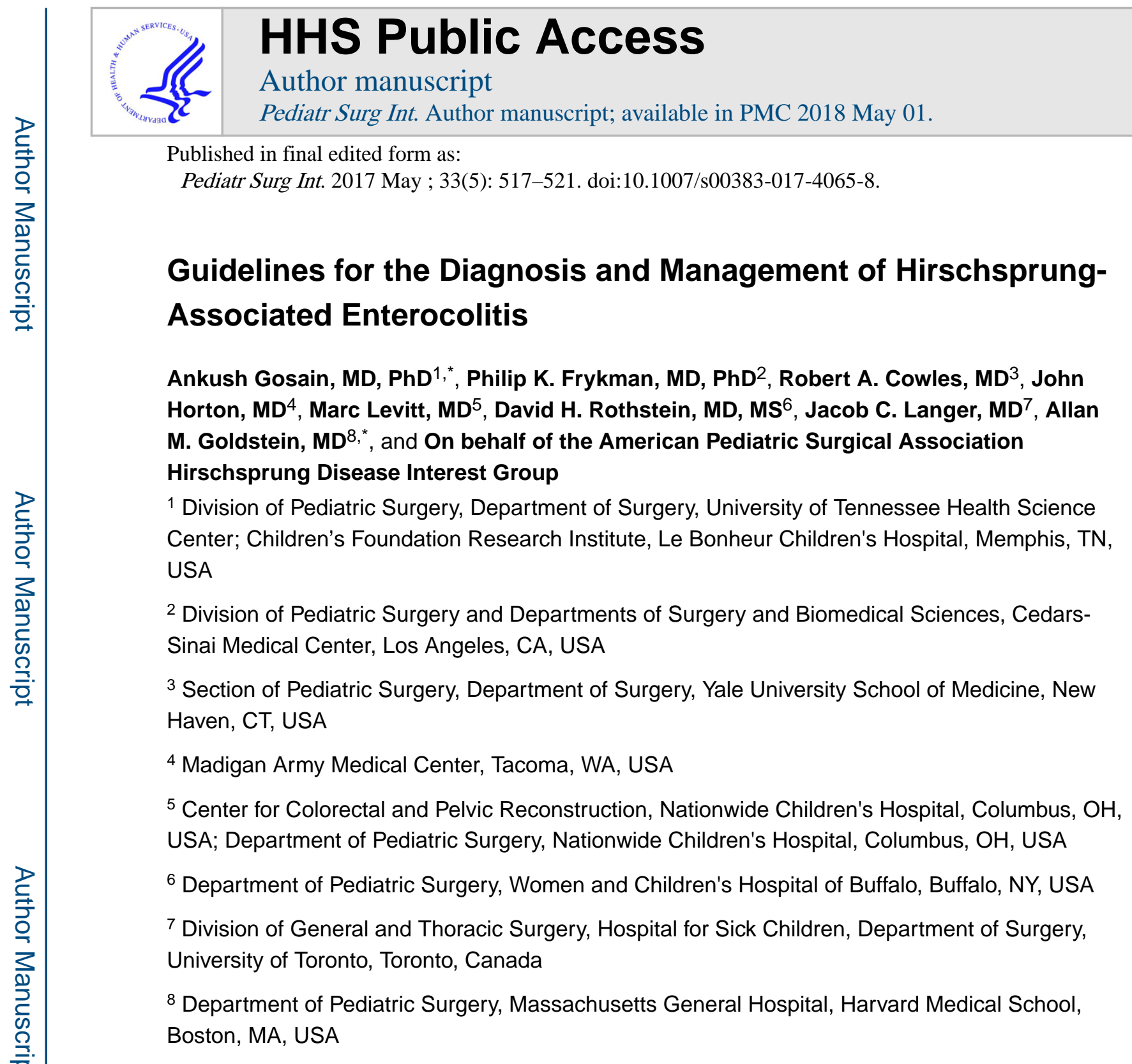

\title{
Abstract
}

Background-Patients with Hirschsprung disease are at risk for Hirschsprung-associated enterocolitis (HAEC), an inflammatory disorder of the bowel that represents the leading cause of serious morbidity and death in these patients. The diagnosis of HAEC is made based on clinical signs and symptoms which are often non-specific, making it difficult to establish a definitive diagnosis in many patients. The purpose of this guideline is to present a rational, expert-based approach to the diagnosis and management of HAEC.

\footnotetext{
*Corresponding Author: Ankush Gosain, MD, PhD, FACS, FAAP, Children's Foundation Research Institute, 50 North Dunlap, Suite 320R, Memphis, TN 38105, agosain@uthsc.edu, Allan M. Goldstein, MD, Department of Pediatric Surgery, Massachusetts General Hospital, 55 Fruit Street, Warren 1151, Boston, MA 02114, agoldstein@partners.org.

Author Contributions:

AG, PKF, JCL, and AMG contributed to the conception, design, and writing; all authors contributed to critical editing of the manuscript and its final approval.
} 
Methods-The American Pediatric Surgical Association Board of Governors established a Hirschsprung Disease Interest Group. Group discussions, literature review and expert consensus were then used to summarize the current state of knowledge regarding diagnosis, management and prevention of Hirschsprung-associated enterocolitis (HAEC).

Results-Guidelines for the diagnosis of HAEC and its clinical grade, utilizing clinical history, physical examination findings, and radiographic findings are presented. Treatment guidelines, including patient disposition, diet, antibiotics, rectal irrigations and surgery, are presented.

Conclusions-Clear, standardized definitions of Hirschsprung-associated enterocolitis and its treatment are lacking in the literature. This guideline serves as a first step towards standardization of diagnosis and management.

Type of Study-Review

\section{Level of Evidence-V}

\section{Keywords}

Hirschsprung disease; Hirschsprung's disease; enterocolitis; Hirschsprung-associated enterocolitis; Hirschsprung's-associated enterocolitis

\section{Background}

Patients with Hirschsprung disease are at risk for Hirschsprung-associated enterocolitis (HAEC), an inflammatory disorder of the bowel that represents the leading cause of serious morbidity and death in these patients. The diagnosis of HAEC is made based on clinical signs and symptoms which are often non-specific, making it difficult to establish a definitive diagnosis in many patients. This has resulted in possible over- and under-treatment of patients as well as difficulties in comparing outcomes based on treatment strategies. The purpose of this guideline is to present a rational, expert-based approach to the diagnosis and management of HAEC.

Given the difficulty often encountered in establishing a definitive diagnosis, the reported incidence of HAEC varies widely, ranging from 6-60\% prior to definitive pull-through surgery and from $25-37 \%$ after surgery [1,2]. While all patients with Hirschsprung disease are at risk for HAEC, several features appear to be associated with an increased risk. These include Down syndrome, long-segment aganglionosis, prior HAEC, and obstruction from any cause (retained aganglionosis, transition zone pull-through, dysmotility following pullthrough, anastomotic stricture, twist in the pull-through or tight muscular cuff following the Soave procedure).

\section{Etiology and pathophysiology}

The cause of HAEC is unknown, although several hypotheses have been proposed based on experimental evidence. These include dysbiosis of the intestinal microbiome[3-6], impaired mucosal barrier function[4, 7, 8], altered innate immune responses[9, 10], and bacterial translocation[4, 11]. 


\section{Diagnosis}

The classic manifestations of HAEC include abdominal distention, fever, and diarrhea.

However, there is a broad clinical spectrum with which children present, and other signs or symptoms may include vomiting, rectal bleeding, lethargy, loose stools, and obstipation. Of note, these symptoms are non-specific and this likely contributes to the highly variable incidence of HAEC reported in the literature. Mild cases, manifesting with only fever, mild distension, and diarrhea, present just like viral gastroenteritis, which is very common in young children. Given the difficulty in making a definitive diagnosis, combined with the high morbidity associated with a delayed or missed diagnosis, most pediatric surgeons err on the side of assigning the diagnosis of HAEC and presumptively treating suspected cases. In general, assuming the child has HAEC and initiating treatment is preferable to delaying the diagnosis and having the child present later with more advanced disease.

In an attempt to address the difficulty in establishing the diagnosis of HAEC, a group of 27 gastroenterologists and surgeons participated in a Delphi process, starting with 38 features (history, patient characteristics, physical exam signs, laboratory findings, radiology findings, pathology findings) and iteratively refining this list to 16 items to develop a HAEC score[12]. The resulting tool, although useful for standardizing outcome measures in research studies, is cumbersome for routine use, has not been validated for clinical application, and has not been widely adopted in the clinical setting. More recently, another group described a "clinical grade" for HAEC for use in a prospective trial[2]. This system grades the degree of diarrhea, abdominal distention and systemic manifestations into mild, moderate and severe in order to assign an overall clinical grade. Following a staging system similar to that described by Bell for necrotizing enterocolitis (NEC)[13], and incorporating elements of published HAEC grading systems and clinical experience, we have categorized the clinical suspicion and severity of HAEC into three grades based on history, physical examination, and imaging studies [Figure 1]. The goal of this approach is to create a standardized, clinically relevant, and easy to use system that can be universally adopted to allow a consistent approach to the diagnosis and treatment of HAEC. In general, presence of higher grade findings should prompt providers to err toward assigning the higher grade and initiating the corresponding treatment.

\section{Management}

Since the cause of HAEC is generally unknown, treatment remains empiric and directed toward alleviating acute symptoms as well as managing the factors that may contribute to pathogenesis. Treatment is based on the severity of the clinical presentation, as detailed in Figure 2 .

In cases classified as Grade I (possible HAEC), outpatient management can typically be employed. Treatment in these cases should include oral metronidazole and oral hydration with Pedialyte or other electrolyte-rich solution. Rectal irrigations to wash retained stool out of the colon should be considered in children who have abdominal distension or who are not evacuating fully. They may also be used as a trial to see if irrigation leads to symptomatic 
improvement. Close monitoring is necessary in case symptoms progress to a higher grade of disease.

In more severe cases, Grade II (Definite HAEC), inpatient admission is often necessary. These children are managed with either clear liquids or nothing by mouth, intravenous fluids, and nasogastric decompression if there is significant abdominal distension. Rectal irrigations are very effective, helping to resolve fecal stasis. Metronidazole (oral or parenteral) is used to treat anaerobes, including Clostridium difficile, which has been associated with HAEC. In addition to metronidazole, broad spectrum intravenous antibiotic coverage using either the combination of ampicillin and gentamicin, or piperacillin/ tazobactam, or aztreonam (in the case of penicillin allergy) should be considered.

Children with findings consistent with Grade III (severe) HAEC, particularly with shock, may require admission to an intensive care unit. Bowel rest, intravenous fluid resuscitation, rectal irrigations, and broad-spectrum antibiotics (including metronidazole) are required. Patients who fail to improve may benefit from proximal enteric diversion. Rarely, pneumoperitoneum can occur, which would prompt immediate surgical intervention.

\section{Prevention}

Some authors have advocated for the use of preventive measures in selected patient populations[14]. These measures include routine use of rectal irrigations in the postoperative period, long-term administration of oral metronidazole, and use of probiotic therapy. A recent prospective randomized trial found that 4 weeks of probiotic therapy decreased the incidence and severity of HAEC [15], but more studies are needed.

\section{Recurrent HAEC}

Children with recurrent HAEC should undergo additional evaluation to determine whether a cause can be identified. In these cases, it is important to evaluate for an anatomic or pathologic cause of obstruction. Anatomic problems include anastomotic stricture, a twisted or kinked anastomosis, megarectum, a Duhamel spur or kink, or a tight Soave cuff. Causes of functional obstruction, such as a transition zone pull-through or retained aganglionosis, should also be considered.

Physical examination is typically performed under anesthesia and should include careful assessment for a stricture, presence and function of the anal sphincters, size of the rectal pouch (if present), and presence of a palpable Soave cuff. A contrast enema using a watersoluble agent can identify any mechanical causes of obstruction. Because of risk of perforation, contrast enemas should not be performed during acute HAEC episodes. Additional evaluation includes rectal biopsy to exclude aganglionosis or transition zone pullthrough. Review of pathology slides from the original surgery should exclude transition zone pull-through. If the workup reveals an anatomic etiology for obstructive symptoms and recurrent HAEC, surgical management directed at correcting the defect should be performed. If there is no anatomic or pathologic cause identified, non-relaxation of the internal anal sphincter may be the cause of stasis with obstructive symptoms and recurrent HAEC in some patients, and can be confirmed by anorectal manometry. Injection of 
Clostridium botulinum toxin (Botox, Allergan, Plc) into the intersphincteric groove has been shown to decrease hospital admissions in children with recurrent symptomatology[16].

\section{Summary}

This guideline presents expert-based consensus recommendations for establishing the diagnosis of HAEC, grading the severity of disease, preventing and treating the associated symptoms as well as any potential contributing factors. It is of utmost importance that parents and pediatricians be familiar with the signs and symptoms of HAEC so that the child is seen by their pediatric surgeon or gastroenterologist promptly as soon as the diagnosis is entertained. Much progress remains to be made in identifying the causes of HAEC and developing effective preventative strategies. We hope that this guideline will help the practicing clinician in the care of these children.

\section{References}

1. Haricharan RN, Seo J-M, Kelly DR, et al. Older age at diagnosis of Hirschsprung disease decreases risk of postoperative enterocolitis, but resection of additional ganglionated bowel does not. J Pediatr Surg. 2008; 43:1115-1123. doi: 10.1016/j.jpedsurg.2008.02.039. [PubMed: 18558193]

2. El-Sawaf M, Siddiqui S, Mahmoud M, et al. Probiotic prophylaxis after pullthrough for Hirschsprung disease to reduce incidence of enterocolitis: A prospective, randomized, double-blind, placebo-controlled,multicenter trial. J Pediatr Surg. 2013; 48:111-117. doi: 10.1016/j.jpedsurg. 2012.10.028. [PubMed: 23331802]

3. Ward NL, Pieretti A, Dowd SE, et al. Intestinal aganglionosis is associated with early and sustained disruption of the colonic microbiome. Neurogastroenterology \& Motility. 2012; 24:874-e400. doi: 10.1111/j.1365-2982.2012.01937.x. [PubMed: 22626027]

4. Pierre JF, Barlow-Anacker AJ, Erickson CS, et al. Intestinal dysbiosis and bacterial enteroinvasion in a murine model of Hirschsprung's disease. J Pediatr Surg. 2014; 49:1242-1251. doi: 10.1016/ j.jpedsurg.2014.01.060. [PubMed: 25092084]

5. Yan Z, Poroyko V, Gu S, et al. Biochemical and Biophysical Research Communications. Biochemical and Biophysical Research Communications. 2014; 445:269-274. doi: 10.1016/j.bbrc. 2014.01.104. [PubMed: 24525127]

6. Frykman PK, Nordenskjöld A, Kawaguchi A, et al. Characterization of Bacterial and Fungal Microbiome in Children with Hirschsprung Disease with and without a History of Enterocolitis: A Multicenter Study. PLoS ONE. 2015; 10:e0124172. doi: 10.1371/journal.pone.0124172. [PubMed: 25909773]

7. Thiagarajah JR, Yildiz H, Carlson T, et al. Altered Goblet Cell Differentiation and Surface Mucus Properties in Hirschsprung Disease. PLoS ONE. 2014; 9:e99944. doi: 10.1371/journal.pone. 0099944.t001. [PubMed: 24945437]

8. Yildiz HM, Carlson TL, Goldstein AM, Carrier RL. Mucus Barriers to Microparticles and Microbes are Altered in Hirschsprung's Disease. Macromol Biosci. 2015; 15:712-718. doi: 10.1002/mabi. 201400473. [PubMed: 25644515]

9. Gosain A, Barlow-Anacker AJ, Erickson CS, et al. Impaired Cellular Immunity in the Murine Neural Crest Conditional Deletion of Endothelin Receptor-B Model of Hirschsprung's Disease. PLoS ONE. 2015; 10:e0128822. doi: 10.1371/journal.pone.0128822. [PubMed: 26061883]

10. Frykman PK, Cheng Z, Wang X, Dhall D. Enterocolitis causes profound lymphoid depletion in endothelin receptor B- and endothelin 3-null mouse models of Hirschsprung-associated enterocolitis. Eur J Immunol. 2015; 45:807-817. doi: 10.1002/eji.201444737. [PubMed: 25487064]

11. Cheng Z, Dhall D, Zhao L, et al. Murine model of Hirschsprung-associated enterocolitis.I: Phenotypic characterization with development of ahistopathologic grading system. J Pediatr Surg. 2010; 45:475-482. doi: 10.1016/j.jpedsurg.2009.06.009. [PubMed: 20223308] 
12. Pastor AC, Osman F, Teitelbaum DH, et al. Development of a standardized definition forHirschsprung's-associated enterocolitis:a Delphi analysis. J Pediatr Surg. 2009; 44:251-256. doi: 10.1016/j.jpedsurg.2008.10.052. [PubMed: 19159752]

13. Bell MJ, Ternberg JL, Feigin RD, et al. Neonatal necrotizing enterocolitis. Therapeutic decisions based upon clinical staging. Ann Surg. 1978; 187:1-7. [PubMed: 413500]

14. Frykman PK, Short SS. Hirschsprung-associated enterocolitis: prevention and therapy. YSPSU. 2012; 21:328-335. doi: 10.1053/j.sempedsurg.2012.07.007.

15. Wang X, Li Z, Xu Z, et al. Probiotics prevent Hirschsprung's disease-associated enterocolitis: a prospective multicenter randomized controlled trial. Int J Colorectal Dis. 2015; 30:105-110. doi: 10.1007/s00384-014-2054-0. [PubMed: 25370155]

16. Patrus B, Nasr A, Langer JC, Gerstle JT. Intrasphincteric botulinum toxin decreases the rate of hospitalization for postoperative obstructive symptoms in children with Hirschsprung disease. J Pediatr Surg. 2011; 46:184-187. doi: 10.1016/j.jpedsurg.2010.09.089. [PubMed: 21238663] 


\begin{tabular}{|c|c|c|c|c|}
\hline Grade & Description & Clinical History & Physical Examination & Radiographic Findings \\
\hline 1 & Possible HAEC & $\begin{array}{l}\text { Anorexia } \\
\text { Diarrhea }\end{array}$ & Mild abdominal distention & $\begin{array}{l}\text { Normal } \\
\text { Mild ileus gas pattern }\end{array}$ \\
\hline ॥ & Definite HAEC & $\begin{array}{l}\text { History of past episode of } \\
\text { HAEC } \\
\text { Explosive diarrhea } \\
\text { Fevers } \\
\text { Lethargy }\end{array}$ & $\begin{array}{l}\text { Fever } \\
\text { Tachycardia } \\
\text { Abdominal distention } \\
\text { Abdominal tenderness } \\
\text { Explosive gas/stool on DRE }\end{array}$ & $\begin{array}{l}\text { Ileus gas pattern } \\
\text { Air/fluid levels } \\
\text { Dilated loops of bowel } \\
\text { Recto-sigmoid cutoff }\end{array}$ \\
\hline III & Severe HAEC & $\begin{array}{l}\text { Obstipation } \\
\text { Obtunded }\end{array}$ & $\begin{array}{l}\text { Decreased peripheral perfusion } \\
\text { Hypotension } \\
\text { Altered mentation } \\
\text { Marked abdominal distention } \\
\text { Peritonitis }\end{array}$ & $\begin{array}{l}\text { Pneumatosis } \\
\text { Pneumoperitoneum }\end{array}$ \\
\hline
\end{tabular}

Figure 1. Guideline for the diagnosis of HAEC

Guideline for the diagnosis and grading of HAEC from grade I (possible HAEC) through grade III (severe HAEC) based on clinical history, physical examination, and radiographic findings. This figure is not intended as a scoring system, but rather a decision-support tool to ensure that all of the relevant history, examination and radiographic findings are considered. In general, presence of higher grade findings should prompt providers to assign the higher grade. (HAEC: Hirschsprung-associated enterocolitis, DRE: digital rectal examination). 


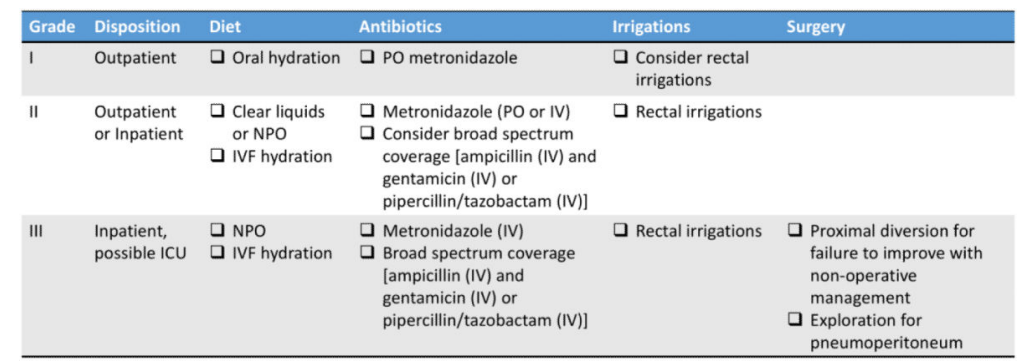

Figure 2. Guideline for the management of HAEC

Guideline for the management of HAEC based on grade. Considerations for diet, antibiotics, rectal irrigations, and need for surgery are listed by grade. (PO: per os, NPO: nothing per os, ICU: intensive care unit, IV: intravenous, IVF: intravenous fluid). 